\title{
As reformas dos sistemas de saúde da América Latina: influências neoliberais e desafios aos Objetivos de Desenvolvimento Sustentável
}

\author{
Health systems reforms in Latin America: neoliberal influences \\ and challenges to the Sustainable Development Goals
}

Leila Bernarda Donato Göttems (https://orcid.org/0000-0002-2675-8085) ${ }^{1}$

Luciano de Paula Camilo (https://orcid.org/0000-0002-4005-7453) ${ }^{2}$

Céline Mavrot (https://orcid.org/0000-0001-9603-5790) ${ }^{3}$

Maria de Lourdes Rollemberg Mollo (https://orcid.org/0000-0002-4303-6914) ${ }^{4}$
${ }^{1}$ Programa de Pós-

Graduação em Ciências para a Saúde, Escola Superior

de Ciências da Saúde. Setor Médico Hospitalar Norte, Asa Norte. 70710-907 Brasília DF Brasil.

leila.gottems@gmail.com

${ }^{2}$ Programa de Pós-

Graduação em Ciências da Saúde, Universidade de Brasília. Brasília DF Brasil

${ }^{3}$ Yale University School of Public Health. New Haven CT EUA.

${ }^{4}$ Departamento de

Economia, Universidade de Brasília. Brasília DF Brasil.

\begin{abstract}
This study analyzes the characteristics of health system reforms in Latin American and Caribbean (LAC) countries, the trend of public health spending, and the achievement of the Millennium Development Goals (MDGs). It also discusses the neoliberal influences on public health reforms and the possible consequences for the upcoming Sustainable Development Goals (SDGs). The study is a comparative, non-exhaustive literature review of selected countries, with data extracted from CEPALStat, Global Health Observatory, MDG Indicators platforms, and the Health in the Americas reports available in the Institutional Repository for Information Sharing of the Pan American Health Organization. The reforms were divided into three periods, namely: up to 1990, with a prevailing regulated national solidarity logic; 1990-2000, moving towards a market-oriented competitive logic; 2001-2015, evolving towards public logic programs, maintaining competition between service providers. Public spending fluctuated over time, and the $M D G$ targets analyzed were not completely met. Changes in health systems followed the models prescribed by neoliberalism, with market-oriented competitive logic, weakening the care system and the achievement of the SDGs.
\end{abstract}

Key words Health system, Health reform, Latin America; Millennium Development Goals
Resumo Analisam-se as características das reformas dos sistemas de saúde de países da América Latina e Caribe (ALC), a evolução dos gastos públicos e dos Objetivos de Desenvolvimento do Milênio (ODM). Discutem-se as influências neoliberais nas reformas e as possiveis consequências para os Objetivos de Desenvolvimento Sustentável (ODS) que os sucederam. Estudo comparado de países selecionados. Dados extraídos das plataformas CEPALStat, Global Health Observatory e MDG Indicators e de relatorios Health in the Americas disponiveis no Repositorio Institucional para Compartir Información da Organização Panamericana de Saúde. Revisão não exaustiva de literatura. As reformas foram divididas em três periodos: até 1990 predominou a lógica nacional solidária regulada; de 1990 a 2000 avançou-se para uma lógica concorrencial de mercado; de 2001 a 2015, evoluiu-se para programas de lógica pública, mantendo-se a concorrência entre prestadores de serviços. Os gastos públicos oscilaram e as metas dos ODM analisadas não foram completamente cumpridas. As mudanças dos sistemas de saúde seguiram as configurações dos Estados nacionais apregoadas pelo neoliberalismo, com lógica concorrencial de mercado, fragilizando o sistema de cuidados e o alcance dos ODS.

Palavras-chave Sistema de saúde, Reforma da saúde, América Latina; Objetivos de Desenvolvimento do Milênio 


\section{Introdução}

Este artigo indaga se o cunho neoliberal das reformas dos sistemas de saúde da década de 1990, em países da América Latina e Caribe (ALC), podem explicar o cumprimento incompleto dos Objetivos do Desenvolvimento do Milênio (ODM) e apontar desafios para o alcance dos Objetivos de Desenvolvimento Sustentável (ODS).

Os ODM foram definidos em 2000, em assembleia da Organização das Nações Unidas (ONU), como compromissos dos 191 países que a integram, com metas estabelecidas para realização até $2015^{1-3}$. A partir daí, entra em cena a Agenda pós-2015, expressa nos Objetivos de Desenvolvimento Sustentável (ODS), que aspiram transcender até 2030 os ODM, ao incorporar outras dimensões nas políticas de saúde ${ }^{2-4}$.

Os ODM/ODS, embora representem um compromisso com resultados de assistência à saúde, homogeneízam as políticas de saúde, com um pacote básico de atenção à saúde reprodutiva voltado para algumas doenças infecto contagiosas, para evitar possíveis efeitos colaterais negativos, e materializam estratégias de organismos internacionais comprometidos com a agenda neoliberal de criação e expansão de mercados, sobretudo de seguros de saúde. Fundamentamse na adoção da cobertura universal da saúde (CUS), concepção compatível com o neoliberalismo econômico, pautando-se na saúde como mercadoria, com intervenção reduzida do Estado. Este fica restrito à regulação do sistema separando-se as funções de financiamento e compra de serviços. Em contraposição, os sistemas universais de saúde, pautados no bem-estar social, têm o Estado como responsável pelo financiamento, gestão e prestação dos serviços de saúde, assim como pela saúde como direito universal ${ }^{4-5}$.

O espaço territorial e geopolítico denominado ALC contempla um conjunto de 20 países muito diversos em termos de história, cultura, características sociodemográficas e econômicas. Apresenta similitudes em termos de mudanças radicais de suas características originais, nos processos de colonização, emigração, imigração e de intercâmbio comercial e tecnológico ${ }^{6}$. Seu processo de desenvolvimento econômico e social tem se caracterizado por ciclos, com diferentes consequências políticas, sociais e estruturais em cada país.

Os anos 1980 são os da crise da dívida da ALC que levou os países em todo o continente à inadimplência em empréstimos, começando com o México em 1982, e se espalhando por todo o continente ${ }^{6-10}$. Em outubro de 1983, 27 países de ren- da baixa e média, muitos nas Américas, estavam inadimplentes em seus empréstimos, ou estavam em processo de reescalonamento de dívidas ${ }^{6}$. Nesse período, o Fundo Monetário Internacional (FMI) e o Banco Mundial (BM) foram chamados a fornecer empréstimos aos países devedores. Associadas a esses empréstimos havia recomendações (condicionalidades) para a implementação de medidas econômicas destinadas a abrir os mercados e atender às prescrições neoliberais do chamado Consenso de Washington. As reformas propostas incluíram cortes drásticos nos gastos do governo (particularmente em saúde e outros setores sociais) e políticas que desmontavam o estado de bem-estar social frágil e incompletamente implantado na $\mathrm{ALC}^{9-11}$.

Para o setor saúde, a agenda dos ajustes econômicos propostos pelos organismos internacionais, sobretudo o Banco Mundial ${ }^{12}$, colocaram-se na contramão do que havia nos países desenvolvidos. Pregavam a redução da responsabilidade do Estado na saúde, devendo se concentrar nos serviços que atendiam a todos, restringindo-se a alguns itens básicos tais como vacinas e controle de doenças de transmissão vetorial. Recomendavam a imposição de encargos aos usuários do sistema público de saúde nos serviços de atenção curativa; estímulo à prestação de serviços de saúde por instituições não governamentais e privadas; descentralização dos serviços de saúde para governos locais, com autonomia financeira, administrativa, de planejamento, orçamento e execução dos serviços de saúde pública; e criação de sistema de pagamento antecipado, como seguro obrigatório de saúde ${ }^{13-15}$.

Tratava-se de recomendações que implicavam em redução do gasto público, principalmente o nacional, abertura econômica à competição internacional, liberalização de preços e medidas para melhorar a eficiência econômica do gasto público e o bom funcionamento da economia de mercado. Confiava-se que as condições de saúde melhorariam no longo prazo como resultado de maior crescimento econômico, e que os primeiros frutos começariam pelo menos cinco anos mais tarde $\mathrm{e}^{14-16}$.

As reformas deveriam distinguir os bens públicos de saúde e garantir os serviços de alta externalidade positiva; considerar o financiamento público da assistência clínica essencial como forma de alívio da pobreza; descentralizar e ajustar o hiper dimensionado sistema de saúde público, limitando novos investimentos em hospitais públicos de atenção terciária, definindo pacote de serviços clínicos essenciais e assegurando os 
serviços básicos conforme recomendação de Alma-Ata desde $1978^{9-11,13-15}$. Ou seja, focalizava-se a demanda a ser assistida pelo poder público, principalmente o nacional, reduzindo despesas por meio da transferência para governos locais e iniciativas não governamentais e privadas, atendendo aos ditames do neoliberalismo ${ }^{16}$.

A transição do modelo econômico centrado no Estado de Bem-Estar Social, ainda que incompletamente implantado na ALC, para um modelo de Estado neoliberal, disseminado nos anos 1970 e 1980, reduziu os sistemas estatais de proteção social e atribuiu ao mercado o atendimento das necessidades sociais, redundando em uma deterioração da qualidade de vida das populações ${ }^{9-10}$. Na década de 2000, com a chamada "onda rosa" da ALC, começaram a ser retomados mecanismos de regulação econômica e proteção social pelos países, desencadeando um "ciclo social" que permitiu que a saúde tivesse papel proeminente, o que se refletiu na definição de três entre oito ODM ${ }^{2,13-15}$.

O período que nos interessa aqui avaliar é o do aprofundamento do neoliberalismo na região. É particularmente na década de 1980, mas principalmente 1990, que esse processo ocorre na ALC. Este artigo analisa as principais características das reformas dos sistemas de saúde realizadas na ALC, a evolução dos gastos públicos em saúde e o cumprimento dos ODM, de forma a refletir sobre as influências neoliberais nas reformas destes sistemas, e os desafios no alcance dos ODS. Assume-se que a dominação do neoliberalismo caracterizou as reformas realizadas na América Latina, levando à excessiva focalização dos serviços de saúde, a desconfiguração dos sistemas e das políticas de saúde pública como resultado da mercantilização e financeirização do setor.

\section{Método}

Realizou-se estudo comparado dos sistemas de saúde de países selecionados da ALC, da evolução dos gastos públicos, discutindo características neoliberais das reformas, buscando-se possíveis relações com as dificuldades para o cumprimento das metas acordadas nos ODM.

Foram selecionados os países com maior população e para cuja análise dos indicadores de saúde havia dados disponíveis: Argentina, Brasil, Chile, Colômbia, México e Peru. Excluiu-se a Venezuela pelos problemas estruturais que tem passado nos últimos anos, que poderiam comprometer os resultados. Cuba entra como con- trafactual na análise das reformas, observando resultados quando a lógica não é a de mercado.

Os dados estatísticos foram extraídos de: CEPALStat (https://estadisticas.cepal.org/cepalstat/portada.html), Global Health Observatory data repository (https://www.who.int/data/gho) e MDG Indicators (Website/http://mdgs.un.org/ unsd/mdg/Data.aspx). Foram feitas análises de relatórios Health in the Americas disponíveis no Repositório Institucional para Compartir Información da Organização Panamericana de Saúde (https://iris.paho.org/xmlui). A revisão de literatura não foi exaustiva.

A partir destes dados e da bibliografia relativa aos sistemas de saúde da ALC foram sintetizados os principais traços característicos dos sistemas de saúde anteriores a 1990, comparando-os com os que resultaram das reformas neoliberais nos anos 1990 e com os existentes mais recentemente após os anos 2000 (Quadro 1). Além disso, foi examinada a evolução dos gastos públicos em saúde (Tabela 1) e o alcance do cumprimento das metas para alcançar os ODM (Tabelas 2 e 3 ).

\section{Resultados}

\section{Processos de reformas dos sistemas de saúde dos países selecionados}

O Quadro 1 mostra a evolução dos sistemas de saúde da ALC desde o período anterior a 1990, quando as reformas ainda não haviam sido realizadas e o neoliberalismo ainda não havia se instalado efetivamente na região, passando pelos anos 1990 até os anos 2000, onde se instalou definitiva e amplamente o receituário neoliberal.

Conforme é possível acompanhar no Quadro 1, o período anterior aos anos 1980 contava com predominância de sistemas nacionais, solidários e regulados. É o caso das Obras Sociais evoluindo para o Instituto Nacional das Obras Sociais na Argentina e do Instituto Nacional de Previdência Social que evoluiu para o Sistema Único de Saúde (SUS), no Brasil. Veja-se também o Serviço Nacional de Saúde no Chile, o Sistema Nacional de Saúde na Colômbia, a Fundação Mexicana de Saúde (FUSALUD), o Instituto Nacional de Saúde Pública (INSP) no México e o Instituto de Previdência Social Peruana.

Nos anos 1990, observa-se uma tendência generalizada na direção de uma lógica de mercado. As preocupações fiscais levaram à redistribuição da prestação de serviços dos governos nacionais para os locais, e à entrega para a iniciativa 
Quadro 1. Contexto histórico e trajetória dos países da ALC de 1990 a 2015.

\begin{tabular}{|c|c|c|c|}
\hline & Até 1990 & $1990-2000$ & 2001-2015 \\
\hline País & $\begin{array}{c}\text { Predomínio da lógica nacional } \\
\text { solidária regulada }\end{array}$ & $\begin{array}{l}\text { Avanço da lógica concorrencial de } \\
\text { mercado }\end{array}$ & $\begin{array}{l}\text { Programas específicos de lógica pública } \\
\text { e comunitária com manutenção da } \\
\text { concorrência de prestadores de serviços }\end{array}$ \\
\hline $\begin{array}{l}\text { A } \\
\text { r } \\
\text { g } \\
\text { e } \\
\text { n } \\
\text { t } \\
\text { i } \\
\text { n } \\
\text { a }\end{array}$ & $\begin{array}{l}1944 \text { - Criação das Obras } \\
\text { Sociais; } 1977 \text { - Extensão das } \\
\text { obras sociais para familiares } \\
\text { dos trabalhadores; } 1984 \text { - 75\% } \\
\text { da população coberta pela } \\
\text { expansão das obras sociais }\end{array}$ & $\begin{array}{l}1990 \text { - Liberalização comercial, } \\
\text { privatização, preocupações fiscais, } \\
\text { cortes de financiamento, províncias } \\
\text { responsáveis pela prestação de } \\
\text { serviços de saúde } \\
1995 \text { - Introdução do setor de } \\
\text { seguros de saúde, escolha pelo } \\
\text { trabalhador } \\
\text { 1999-2002 - Baixa capacidade de } \\
\text { resposta das Obras Sociais diante de } \\
\text { crise econômica }\end{array}$ & $\begin{array}{l}\text { 2003-2007 - Ajustes no sistema misto de } \\
\text { pensões público-privadas e Plano Federal de } \\
\text { Saúde: política farmacêutica nacional, saúde } \\
\text { materno-infantil, seguro de saúde pública, } \\
\text { programas específicos e APS } \\
2008 \text { - 90\% de cobertura da população com a } \\
\text { renacionalizarão e unificação do sistema misto } \\
\text { de pensões público-privadas } \\
\text { 2011- Lei de Medicina Pré-Paga } \\
\text { 2015- Sistema de saúde segmentado, } \\
\text { fragmentado, com baixos níveis de eficiência } \\
\text { e igualdade. Serviços nacionais, provinciais e } \\
\text { municipais }\end{array}$ \\
\hline $\begin{array}{l}\text { B } \\
\mathrm{r} \\
\mathrm{a} \\
\mathrm{s} \\
\mathrm{i} \\
\mathrm{l}\end{array}$ & $\begin{array}{l}\text { Séc. XX - Foco no controle } \\
\text { de doenças específicas e em } \\
\text { segmento de trabalhadores } \\
1960-1970 \text { - Subsídios estatais } \\
\text { ao setor privado e expansão } \\
\text { da cobertura da assistência } \\
\text { médica previdenciária para } \\
\text { trabalhadores rurais } \\
1974 \text { - Sistema Nacional de } \\
\text { Saúde } \\
1988 \text { - Nova Constituição } \\
\text { Federal define saúde como } \\
\text { direito e cria o SUS }\end{array}$ & $\begin{array}{l}\text { 1995-2002- Descentralização de } \\
\text { governo federal para os municípios. } \\
\text { Programa Saúde da Família, saúde } \\
\text { mental, controle de HIV/AIDS e } \\
\text { controle do tabaco } \\
1998 \text { - Regulação do subsetor } \\
\text { privado (Lei de regulação) } \\
2000 \text { - Criação da Agência Nacional } \\
\text { de Saúde Suplementar }\end{array}$ & $\begin{array}{l}2003 \text { a } 2014 \text { - Fortalecimento da APS/ ESF; } \\
\text { Programa Mais Médicos, saúde bucal, atenção } \\
\text { às urgências e assistência farmacêutica; } \\
\text { produção nacional de insumos estratégicos } \\
\text { para a saúde, educação e gestão do trabalho em } \\
\text { saúde e regulação dos planos e seguros de saúde } \\
2011 \text { - Decreto } 7508 \text { regulamentando LOS no } \\
8.080 / 1990 \\
\text { 2012 - Lei } 142 \text { regulamenta a EC no } 29 \text { com } \\
\text { definição de percentuais mínimos para estados } \\
\text { e municípios aplicarem na saúde } \\
\text { 2014-2015- Medida Provisória e Lei autorizam } \\
\text { abertura do setor saúde ao capital estrangeiro }\end{array}$ \\
\hline $\begin{array}{l}\text { C } \\
\text { h } \\
\text { i } \\
\text { l } \\
\text { e }\end{array}$ & $\begin{array}{l}1924 \text { - Criação do Ministério } \\
\text { de Higiene, Assistência Social e } \\
\text { Trabalho } \\
1948 \text { - Criação da Associação } \\
\text { Médica Chilena } \\
1952 \text { - Lei estabelecendo seguro } \\
\text { obrigatório contra os riscos } \\
\text { de doença, invalidez, velhice e } \\
\text { morte e cria o SNS } \\
\text { 1980 - Ditadura militar, } \\
\text { privatização do asseguramento } \\
\text { e da atenção médica }\end{array}$ & $\begin{array}{l}1990 \text { - Criam-se o Fondo Nacional } \\
\text { de Salud (FONASA) financiado por } \\
\text { recursos públicos e cotizações dos } \\
\text { beneficiários. O Institutos de Salud } \\
\text { Previsionales (Isapres) é criado } \\
1995 \text { - Estatuto da APS Lei no } 19.378 \\
2000-2006 \text { - Fortalecimento do } \\
\text { sistema público, com configuração } \\
\text { dual }\end{array}$ & $\begin{array}{l}2003 \text { - Lei do Financiamento No } 19.888: \\
\text { aumento do valor do IVA } \\
2005 \text { - Lei das Instituciones de Salud Previsional } \\
\text { - ISAPRE no } 20.015 \\
2012 \text { - Lei de Direitos e Deveres em Saúde no } \\
20.584 \\
2017 \text { - Orientações para a Planificação e } \\
\text { Programação em Rede, baseadas na lógica das } \\
\text { RISS }\end{array}$ \\
\hline $\begin{array}{c}\text { C } \\
\mathbf{o} \\
\mathbf{1} \\
\hat{\mathbf{o}} \\
\mathbf{m} \\
\mathbf{b} \\
\mathbf{i} \\
\mathbf{a}\end{array}$ & $\begin{array}{l}1975 \text { - SNS de nível nacional, } \\
\text { departamental e municipal, } \\
\text { integrando os subsetores } \\
\text { público, privado e a seguridade } \\
\text { social além do Instituto } \\
\text { Colombiano de Seguros } \\
\text { Sociales (ICSS) } \\
1977-1980 \text { - Leis no } 1.650, \\
\text { 1.651, } 1.652 \text { e } 1.653 \text { de } 1977, \\
\text { convertem o ICSS em Instituto } \\
\text { de Seguros Sociales (ISS) com } \\
\text { medidas de descentralização } \\
\text { administrativa }\end{array}$ & $\begin{array}{l}1990 \text { - Lei } 10 \text { que delimitou poderes } \\
\text { e responsabilidades nacionais } \\
\text { e territoriais da nação, dos } \\
\text { departamentos e municípios, em } \\
\text { termos de gestão da saúde } \\
1991 \text { a } 1993 \text { - CF e Leis no } 60 \\
\text { e no } 100 \text {, definem recursos e } \\
\text { responsabilidades dos territórios na } \\
\text { saúde, unificação das contribuições } \\
\text { obrigatórias e planos de benefícios e } \\
\text { novas fontes de recursos: impostos } \\
\text { específicos e participação nas } \\
\text { receitas do petróleo }\end{array}$ & $\begin{array}{l}\text { 2000-2015 - A estrutura e organização dos } \\
\text { serviços de saúde aplicam as diretrizes da Lei } \\
\text { 100/1993 } \\
2001 \text { - Regulamentação do processo de } \\
\text { descentralização por meio da Lei 715/2001 } \\
2007 \text { - A Lei } 1.122 \text { introduziu ajustes no sistema } \\
\text { de saúde colombiano para promover o acesso } \\
\text { universal } \\
\text { 2015- O Congresso da República promulgou a } \\
\text { Lei Estatutária } 1.751\end{array}$ \\
\hline
\end{tabular}


Quadro 1. Contexto histórico e trajetória dos países da ALC de 1990 a 2015.

\begin{tabular}{|c|c|c|c|}
\hline & Até 1990 & $1990-2000$ & 2001-2015 \\
\hline País & $\begin{array}{c}\text { Predomínio da lógica nacional } \\
\text { solidária regulada }\end{array}$ & $\begin{array}{l}\text { Avanço da lógica concorrencial de } \\
\text { mercado }\end{array}$ & $\begin{array}{l}\text { Programas específicos de lógica pública } \\
\text { e comunitária com manutenção da } \\
\text { concorrência de prestadores de serviços }\end{array}$ \\
\hline $\begin{array}{c}\mathbf{M} \\
\text { é } \\
\mathbf{x} \\
\mathbf{i} \\
\mathbf{c} \\
\mathbf{o}\end{array}$ & $\begin{array}{l}\text { 1943-1959 - Criação do } \\
\text { Instituto Mexicano de } \\
\text { Seguridade Social (IMSS) } \\
\text { e Instituto de Seguridade } \\
\text { e Serviços Sociais dos } \\
\text { Trabalhadores do Estado } \\
\text { (ISSSTE) } \\
1983 \text { - Constituição reconhece } \\
\text { o direito à proteção social à } \\
\text { saúde } \\
\text { 1982-1988 - Reforma com } \\
\text { ênfase na racionalização, } \\
\text { descentralização e } \\
\text { diversificação dos prestadores } \\
\text { de serviços. Criou-se a } \\
\text { Fundação Mexicana de Saúde } \\
\text { (FUNSALUD), e o Instituto } \\
\text { Nacional de Saúde Pública } \\
\text { (INSP) }\end{array}$ & $\begin{array}{l}1995 \text { - Início da segunda onda } \\
\text { de descentralização para ampliar } \\
\text { responsabilidades estaduais no } \\
\text { financiamento, gestão e prestação de } \\
\text { serviços }\end{array}$ & $\begin{array}{l}\text { 2000-2015 - A estrutura e organização dos } \\
\text { serviços de saúde aplicam as diretrizes da Lei } \\
\text { 100/1993, que instituiu a descentralização e a } \\
\text { seguridade } \\
2001 \text { - Regulamentação do processo de } \\
\text { descentralização por meio da Lei 715/2001 } \\
\text { que redefiniu o fluxo de recursos financeiros e } \\
\text { competências municipais } \\
2007 \text { - A Lei } 1122 \text { introduziu ajustes no sistema } \\
\text { de saúde colombiano para promover o acesso } \\
\text { universal } \\
\text { 2015- O Congresso da República promulgou a } \\
\text { Lei Estatutária } 1.751\end{array}$ \\
\hline $\begin{array}{l}\text { C } \\
\mathbf{u} \\
\mathbf{b} \\
\mathbf{a}\end{array}$ & $\begin{array}{l}\text { Anos } 1960 \text { - CF referenda os } \\
\text { princípios humanistas e de } \\
\text { solidariedade da Saúde Pública, } \\
\text { construção da rede de serviços, } \\
\text { da Faculdade de Medicina, do } \\
\text { Ministério da Saúde e indústria } \\
\text { farmacêutica } \\
1961 \text { - Criação de SNS } \\
\text { integrado, com financiamento } \\
\text { fiscal total, acesso universal e } \\
\text { atenção gratuita } \\
1963 \text { - Cooperação Médica } \\
\text { Internacional } \\
1975 \text { - Atenção à saúde a todos } \\
\text { os cidadãos na Constituição }\end{array}$ & $\begin{array}{l}1990 \text { - O Sistema de Saúde Cubano } \\
\text { enfatiza: Cuidados de Saúde } \\
\text { Primários }\end{array}$ & $\begin{array}{l}2001 \text { - Cuba realiza cooperações internacionais } \\
\text { com diversos países } \\
2002 \text { - Programas de Revolução em Saúde, } \\
\text { Reformulação da estrutura organizativa } \\
\text { dos serviços de saúde, em particular a APS, } \\
\text { aprofundando o trabalho do médico e } \\
\text { enfermeiro de família } \\
2004 \text { - Policlínico Universitário, como novo } \\
\text { modelo de formação }\end{array}$ \\
\hline $\begin{array}{l}\mathrm{P} \\
\mathrm{e} \\
\mathrm{r} \\
\mathrm{u}\end{array}$ & $\begin{array}{l}1978 \text { - Formação do Sistema } \\
\text { Nacional de Serviços de } \\
\text { Saúde, em torno do Instituto } \\
\text { Previdência Social Peruana } \\
\text { (IPSS), oferecendo serviços de } \\
\text { saúde através da cooperação } \\
\text { com entidades privadas Anos } \\
\text { 1980- dificuldades econômicas } \\
\text { de gestão e baixo aporte } \\
\text { econômico do Estado }\end{array}$ & $\begin{array}{l}1990 \text { - Criação do SNS por Decreto } \\
\text { Legislativo no } 584 \\
\text { 1997- Lei Geral de Saúde criou } \\
\text { sistema dual com acesso universal } \\
\text { na saúde pública e um subsetor } \\
\text { privado; elimina garantia de } \\
\text { financiamento absoluto por parte } \\
\text { do Estado, cria a Instituiciones } \\
\text { Prestadoas de Servicios de Salud - } \\
\text { IPRESS), que gerencia centros de } \\
\text { saúde públicos, privados e mistos } \\
\text { registrados na SUNASA }\end{array}$ & $\begin{array}{l}2002 \text { - Lei no } 27.813 \text { cria o Sistema Nacional } \\
\text { Coordinado y Descentralizado de Salud } \\
\text { (SNCDS) } \\
2009 \text { - Lei } 29344 \text { e seu Decreto regulamentador } \\
\text { redesenharam o sistema com separação } \\
\text { de funções de asseguramento e prestação } \\
\text { de serviços, incorporando mecanismos de } \\
\text { mercado e espaço para atuação do setor } \\
\text { privado } \\
\text { 2015 - Lei } 1751 \text { Marco de Aseguramiento } \\
\text { Universal en Salud - modifica direito } \\
\text { fundamental à saúde e a politica de acesso ao } \\
\text { sistema }\end{array}$ \\
\hline
\end{tabular}

Legenda: EC: Emenda Constitucional; APS: Atenção Primária à Saúde; IVA: Imposto sobre Valor Agregado; RISS: Redes Integradas de Serviços de Saúde; SNS: Serviço Nacional de Saúde; SUNASA: Superintendência Nacional de Seguro Saúde; CF: Constituição Federal.. 
privada das gestões e/ou financiamento, e/ou prestação dos serviços de saúde. É o período de descentralização das responsabilidades, criação de distintas formas de financiamento e organização da prestação privada de serviços de saúde, do crescimento dos planos da saúde privados, da segmentação da cobertura da população e fragmentação no fornecimento dos serviços (seguro popular, social, público, privado etc.). Destaca-se entre os sistemas mais segmentados, e cuja segmentação se iniciou ainda nos anos anteriores a 1990, o caso da Colômbia. O Brasil, neste período, trilhou o caminho inverso, rumo ao Sistema Único de Saúde, de caráter universal, ainda que descentralizando-o para municípios e compartilhando a prestação de serviços de saúde com os planos privados, em um sistema dual.

Depois dos anos 2000, percebe-se um certo retorno às preocupações com a saúde pública, com o aparecimento de programas específicos de lógica pública e comunitária, preocupações com regulação e coordenação, mas mantendo a concorrência entre prestadores de serviços de saúde, garantindo ainda uma lógica de mercado. É o caso da ampliação de programas públicos específicos na Argentina, da regulação dos planos de saúde no Brasil, do aumento de impostos para financiamento da saúde, da regulamentação da liberdade das ISAPRES no Chile, e da expansão dos serviços públicos em alguns estados e do IMSS - Oportunidades para beneficiários do Seguro Popular no México, bem como da Ley Marco de Aseguramiente Universal en Salud no Perú.

Cuba foi analisada como contrafactual. Tratase de um país reconhecidamente bem sucedido em termos de indicadores de saúde, e se percebe sua evolução em direção completamente diferente. De um sistema já público e universal desde o início da análise, evolui na direção do aprofundamento e ampliação da prestação de serviços de saúde e na articulação desta prestação com a formação em saúde, internamente. Externamente, cresce a ampliação da cooperação internacional.

\section{Gastos públicos e privados em saúde e em relação ao PIB}

A Tabela 1 mostra que, com exceção da Argentina, os países aumentaram os gastos em saúde em relação ao PIB, no período de 1980 a 2015. Observa-se, porém, que em todos eles a participação do setor público no provimento de saúde cai entre 1980 - quando o neoliberalismo ainda não havia se generalizado na região - e os anos posteriores, período de expansão maior do neoliberalismo. Em alguns países, como Brasil e Chile, essa redução se estende até os anos 2000. Nos períodos seguintes, que presenciaram a chamada onda rosa de governos de esquerda, os dados de participação do setor público nos gastos de saúde aumentam em todos os países, embora em proporções distintas. Os efeitos da retomada neoliberal, porém, só poderão ser analisados com dados posteriores a 2015, para os quais ainda não se tem informações consolidadas.

Observe-se, a esse respeito, que a retirada dos estados nacionais dos gastos com saúde compromete o acesso a esses serviços. Por um lado, surgem os pacotes diferenciados em termos de preços e, por outro, surgem os planos segmentando o acesso da população por renda, classe e categoria funcional, como vimos nas reformas de saúde do Quadro 1. Isso não apenas exclui ou limita o acesso de alguns, mas, ao fazê-lo, reduz o poder de pressão pela qualidade e quantidade dos serviços, já que trata diferenciadamente os que formam opinião.

\section{Desempenho dos países nos ODM e sua transição para os ODS}

Em relação ao desempenho dos indicadores dos ODM, as Tabelas 2 e 3 mostram que a maioria dos países não alcançou todas as metas acordadas. Quanto à meta de redução em dois terços da mortalidade infantil em menores de 5 anos, no período de 1990 e 2015, somente cinco países da região, sendo três selecionados e estudados neste artigo, alcançaram a meta, que era de redução de 66\%: Brasil (-75,1\%), México (-67,0\%) e Perú $(-80 \%)$. Neste indicador, Cuba e Chile apresentam o melhor desempenho no período: embora não tenham atingido a meta de redução, sua taxa de mortalidade é, de longe, a mais baixa em números absolutos.

Em relação à meta de redução da taxa de mortalidade infantil (TMI) em $66 \%$ entre crianças menores de 1 ano, apenas o Peru (-78\%) e o Brasil $(-73,3 \%)$ atingiram a meta. Chile e Cuba apresentaram novamente o melhor desempenho geral, com a menor TMI desde os anos 1990. Quanto à proporção de crianças de 1 ano imunizadas contra sarampo, Brasil, Colômbia, Cuba, México e Peru alcançaram uma variação positiva final. Os melhores resultados estão no Brasil e Cuba (99\% em 2015). A meta da Razão de Mortalidade Materna (RMM) era alcançar uma redução de três quartos. Embora nenhum dos países tenha atingido essa meta, todos eles reduziram o número de mortes maternas evitáveis, com as 
maiores reduções ocorrendo no Peru (-72,9\%) e no Chile $(-61,4 \%)$. O Chile é o país com a RMM mais baixa $(22 / 100.000 \mathrm{NV})$, seguido pelo México $(38 / 100.000 \mathrm{NV})$.
Quanto ao indicador "proporção de nascimentos assistidos por pessoal de saúde qualificado" (médicos, enfermeiros ou parteiras), de acordo com os dados parcialmente disponíveis, todos

Tabela 1. Distribuição dos gastos totais, públicos e privados como \% do PIB.

\begin{tabular}{|c|c|c|c|c|}
\hline País & Anos & $\%$ do PIB gasto em saúde & $\%$ do gasto público & $\%$ do gasto privado \\
\hline \multirow[t]{7}{*}{ Argentina } & $1985^{1}$ & 8,2 & 65,9 & 34,1 \\
\hline & 1990 & 10,5 & 40,2 & 59,8 \\
\hline & 1995 & 8,2 & 44,5 & 55,5 \\
\hline & 2000 & 8,6 & 55 & 45 \\
\hline & 2005 & 8 & 51 & 49 \\
\hline & 2010 & 9 & 65 & 35 \\
\hline & 2015 & 6.8 & 72 & 28 \\
\hline \multirow[t]{7}{*}{ Brasil } & $1982^{2}$ & 8 & 71,5 & 28,5 \\
\hline & 1990 & 6,6 & 45,9 & 54,1 \\
\hline & 1995 & 7,2 & 48,7 & 51,3 \\
\hline & 2000 & 8,3 & 40,8 & 59,2 \\
\hline & 2005 & 8 & 42 & 58 \\
\hline & 2010 & 8 & 45 & 55 \\
\hline & 2015 & 8,9 & 43 & 57 \\
\hline \multirow[t]{7}{*}{ Chile } & $1980^{1}$ & 7,4 & 60 & 40 \\
\hline & 1990 & 4,8 & 45,6 & 54,4 \\
\hline & 1995 & 6,7 & 46 & 54 \\
\hline & 2000 & 7,2 & 42,6 & 57,4 \\
\hline & 2005 & 7 & 53 & 47 \\
\hline & 2010 & 7 & 59 & 41 \\
\hline & 2015 & 8,1 & 61 & 39 \\
\hline \multirow[t]{7}{*}{ Colômbia } & $1980^{5}$ & 3,9 & 50 & 50 \\
\hline & 1990 & 5,6 & 21,3 & 78,7 \\
\hline & 1995 & 7,4 & 45,1 & 54,1 \\
\hline & 2000 & 9,6 & 55,8 & 44,2 \\
\hline & 2005 & 6 & 72 & 28 \\
\hline & 2010 & 6 & 70 & 30 \\
\hline & 2015 & 6,2 & 66,7 & 29,3 \\
\hline \multirow[t]{7}{*}{ México } & $1982^{4}$ & 2,9 & 54,8 & 45,2 \\
\hline & 1990 & 4,4 & 40,9 & 59,1 \\
\hline & 1995 & 5,6 & 39 & 61 \\
\hline & 2000 & 5,4 & 46,5 & 53,5 \\
\hline & 2005 & 6 & 42 & 58 \\
\hline & 2010 & 6 & 50 & 50 \\
\hline & 2015 & 11 & 52 & 48 \\
\hline \multirow[t]{7}{*}{ Peru } & $1980^{3}$ & 5,6 & 90 & 10 \\
\hline & 1990 & 8,2 & 15,6 & 84,4 \\
\hline & 1995 & 4,6 & 44,5 & 55,5 \\
\hline & 2000 & 4,8 & 59,2 & 40,8 \\
\hline & 2005 & 5 & 55 & 45 \\
\hline & 2010 & 5 & 52 & 48 \\
\hline & 2015 & 5,9 & 62 & 38 \\
\hline
\end{tabular}

Fonte: Dados 1990 e 2000: (1) $\mathrm{PAHO}^{31}$; (2) $\mathrm{PAHO}^{30}$; (3) World Bank,1993 ${ }^{12}$; (4) Jaramillo, 2002 $2^{32}$. Dados de 2005-2015: Global Health Expenditure Database (http://apps.who.int/nha/database/ViewData/Indicators/es). 
Tabela 2. Cumprimento das Metas 4 e 5 dos ODMs - Saúde Materno Infantil.

\begin{tabular}{|c|c|c|c|c|c|c|c|}
\hline Indicador & & $\begin{array}{c}\text { TMI }<5 \\
\text { anos } \\
\text { (por mil) }\end{array}$ & $\begin{array}{c}\text { TMI } \\
<1 \text { anos } \\
\text { (por mil) }\end{array}$ & $\begin{array}{l}\% \text { crianças } \\
\text { imunizadas }\end{array}$ & $\begin{array}{c}\text { MMR } \\
\text { (por } \\
100 \mathrm{mil})\end{array}$ & $\begin{array}{c}\text { Nascimentos } \\
\text { assistidos }\end{array}$ & $\begin{array}{c}\text { Gravidez de } \\
\text { adolescentes } \\
15-19 \text { anos }\end{array}$ \\
\hline Metas & Anos & Reduçã & de $66 \%$ & & $\begin{array}{c}\text { Redução de } \\
75 \%\end{array}$ & $100 \%$ & $\begin{array}{c}\text { Saúde } \\
\text { Reprodutiva } \\
(100 \%)\end{array}$ \\
\hline \multirow{7}{*}{ ARG } & 1990 & 28,6 & 25,3 & 93 & 72 & & \\
\hline & 1995 & 24,1 & 21,5 & 99 & 63 & & \\
\hline & 2000 & 19,6 & 17,5 & 91 & 60 & 99,1 & 64,7 \\
\hline & 2005 & 16,5 & 14,8 & 99 & 58 & 99,1 & 63,4 \\
\hline & 2010 & 14,4 & 12,9 & 98 & 58 & 95 & 68,2 \\
\hline & 2015 & 11,5 & 10,2 & 91 & 52 & 99,6 & 65,1 \\
\hline & Var\% & $-59,8$ & $-59,7$ & $-2,2$ & $-27,8$ & 0,5 & 0,6 \\
\hline \multirow[t]{7}{*}{ BRA } & 1990 & 63 & 52,5 & 78 & 104 & & \\
\hline & 1995 & 47,9 & 41 & 87 & 84 & & \\
\hline & 2000 & 34,8 & 30,4 & 99 & 66 & 98,6 & 81,4 \\
\hline & 2005 & 24,9 & 22,1 & 99 & 67 & 98,6 & 73,9 \\
\hline & 2010 & 18,7 & 16,7 & 99 & 65 & 98,9 & 62,2 \\
\hline & 2015 & 15,7 & 14 & 99 & 44 & 99,1 & 61,7 \\
\hline & Var\% & $-75,1$ & $-73,3$ & 26,9 & $-57,7$ & 0,5 & $-24,2$ \\
\hline \multirow[t]{7}{*}{$\mathrm{CHI}$} & 1990 & 19,1 & 16,1 & 97 & 57 & & \\
\hline & 1995 & 13,1 & 11,1 & 97 & 41 & & \\
\hline & 2000 & 10,9 & 9,2 & 97 & 31 & 99 & 62,7 \\
\hline & 2005 & 9,1 & 7,7 & 90 & 27 & 99,8 & 50,4 \\
\hline & 2010 & 8,7 & 7,4 & 93 & 26 & 99,8 & 53,5 \\
\hline & 2015 & 7,9 & 6,7 & 90 & 22 & 99,7 & 40,6 \\
\hline & Var\% & $-58,6$ & $-58,4$ & $-7,2$ & $-61,4$ & 0,7 & $-35,2$ \\
\hline \multirow[t]{7}{*}{$\mathrm{COL}$} & 1990 & 35,2 & 28,9 & 82 & 118 & & \\
\hline & 1995 & 29,7 & 24,8 & 95 & 105 & & \\
\hline & 2000 & 25 & 21,1 & 88 & 97 & 86,1 & 80,6 \\
\hline & 2005 & 21,5 & 18,3 & 96 & 80 & 90,7 & 96,2 \\
\hline & 2010 & 18,5 & 15,8 & 88 & 72 & 94,8 & $\ldots$ \\
\hline & 2015 & 15,7 & 13,5 & 92 & 64 & 95,9 & $\ldots$ \\
\hline & Var\% & $-55,4$ & $-53,3$ & 12,2 & $-45,8$ & 11,4 & 19,4 \\
\hline
\end{tabular}

continua

os países melhoraram, mas o objetivo global permaneceu inalcançado. Nem todos os países têm dados disponíveis sobre o percentual de mães com idade entre 15-19 anos de idade (ODM5). A Argentina, Colômbia e Cuba apresentam aumentos de na taxa de nascimentos entre adolescentes de 2000 a 2015. Os demais países reduziram o número de nascimentos entre adolescentes, embora os dados de Colômbia, Peru e México mostrem-se muito incompletos.

A meta 6 dos ODM (Tabela 3) teve como objetivo iniciar o processo de reverter a disseminação do HIV/AIDS até 2015. Segundo os dados disponíveis até 2014, o México e o Peru reverteram a propagação de AIDS entre 15-49 anos. Quanto à meta de acesso universal ao tratamento, todos os países expandiram o acesso com destaque para o Brasil (95\%) e Cuba (95\%) que alcançaram os melhores resultados.

Buscou-se também analisar o alcance das metas de reversão da incidência da malária e tuberculose (TB) até 2015. Os dados disponíveis sobre a taxa de incidência associada à malária e à $\mathrm{TB}$ não estavam completamente disponíveis antes de 2000. De acordo com a Tabela 3, todos os países diminuíram a incidência e a prevalência destas doenças infectocontagiosas. 
Tabela 2. Cumprimento das Metas 4 e 5 dos ODMs - Saúde Materno Infantil.

\begin{tabular}{|c|c|c|c|c|c|c|c|}
\hline Indicador & & $\begin{array}{c}\text { TMI }<5 \\
\text { anos } \\
\text { (por mil) }\end{array}$ & $\begin{array}{c}\text { TMI } \\
<1 \text { anos } \\
\text { (por mil) }\end{array}$ & $\begin{array}{l}\text { \% crianças } \\
\text { imunizadas }\end{array}$ & $\begin{array}{c}\text { MMR } \\
\text { (por } \\
100 \mathrm{mil})\end{array}$ & $\begin{array}{c}\text { Nascimentos } \\
\text { assistidos }\end{array}$ & $\begin{array}{c}\text { Gravidez de } \\
\text { adolescentes } \\
15-19 \text { anos }\end{array}$ \\
\hline Metas & Anos & Reduçã & de $66 \%$ & & $\begin{array}{c}\text { Redução de } \\
75 \%\end{array}$ & $100 \%$ & $\begin{array}{c}\text { Saúde } \\
\text { Reprodutiva } \\
(100 \%)\end{array}$ \\
\hline \multirow[t]{7}{*}{ CUB } & 1990 & 13,4 & 10,9 & 94 & 58 & & \\
\hline & 1995 & 10,6 & 8,5 & 99 & 55 & & \\
\hline & 2000 & 8,5 & 6,8 & 94 & 43 & 99,9 & 49,5 \\
\hline & 2005 & 7,2 & 5,6 & 98 & 41 & 99,9 & 43,4 \\
\hline & 2010 & 6,1 & 4,7 & 99 & 44 & 99,9 & 53,6 \\
\hline & 2015 & 5,4 & 4,1 & 99 & 39 & 99,9 & $\ldots$ \\
\hline & Var\% & $-59,7$ & $-62,4$ & 5,3 & $-32,8$ & 0,0 & 8,3 \\
\hline \multirow[t]{7}{*}{ MÉX } & 1990 & 44,8 & 35,9 & 75 & 90 & & \\
\hline & 1995 & 34,7 & 28,6 & 90 & 85 & & \\
\hline & 2000 & 26,4 & 22,2 & 96 & 77 & 88,6 & 89,7 \\
\hline & 2005 & 20,5 & 17,5 & 96 & 54 & $\ldots$ & $\ldots$ \\
\hline & 2010 & 17,4 & 12,7 & 95 & 45 & $\ldots$ & $\ldots$ \\
\hline & 2015 & 14,8 & & 89 & 38 & 97,7 & 65,4 \\
\hline & Var\% & $-67,0$ & $-64,6$ & 18,7 & $-57,8$ & 10,3 & $-27,1$ \\
\hline \multirow[t]{7}{*}{ PER } & 1990 & 80,5 & 56,9 & 64 & 251 & & \\
\hline & 1995 & 58,3 & 43,1 & 98 & 206 & & \\
\hline & 2000 & 38,6 & 29,6 & 97 & 140 & 59,3 & $\ldots$ \\
\hline & 2005 & 26,7 & 20,7 & 77 & 114 & $\ldots$ & 64 \\
\hline & 2010 & 20,1 & 15,6 & 94 & 92 & 83,8 & 61,1 \\
\hline & 2015 & 16,1 & 12,5 & 85 & 68 & 91,6 & $\ldots$ \\
\hline & Var\% & -80 & $-78,0$ & 32,8 & $-72,9$ & 54,5 & $-4,5$ \\
\hline
\end{tabular}

Observação: Variação em \% (Var\%) expressa a diferença entre o primeiro e o último ano analisado.

Fonte: CEPALStat e Global Health Observatory Data Repository.

\section{Discussão}

Os caminhos das reformas em saúde mostraram diferentes aderências às duas concepções opostas. A primeira é a de Cobertura Universal de Saúde, que tem como foco o financiamento por combinação de fundos (pooling), a afiliação por modalidade de asseguramento e a definição de cesta limitada de serviços. Esta foi a forma que predominou sobretudo no México em 1990 e na Colômbia em $1993^{4}$. A segunda é a do Sistema Universal de Saúde, financiado por fundos públicos, a partir da receita de impostos gerais e contribuições sociais, o que proporciona maior solidariedade, redistribuição e equidade ${ }^{4-5}$. Este é o modelo do Brasil, embora mais nos aspectos legais do que reais, uma vez que reparte a prestação de serviços com os planos privados de saúde, configurando um sistema dual. Entre os dois ex- tremos, modelos intermediários variam no nível de acesso e amplitude da cobertura, assim como nas modalidades de afiliação aos seguros e no grau de integração entre o setor público e o setor privado para a prestação de serviços. Neste polo intermediário encontram-se os demais países analisados: Argentina, Peru, Chile ${ }^{4,5,9}$.

Fortaleceu-se nas reformas, a participação privada na gestão e prestação de serviços de saúde ${ }^{11-13-15,33-34}$. Produziram-se serviços de saúde descentralizados e segmentados ${ }^{26}$, quanto ao acesso e ao tipo de serviço fornecido, e fragmentado (dual, tripartite e até quadripartite) quanto ao planejamento e à gestão de recursos entre os prestadores dos serviços, com esquemas de copagamento, ou seja, pagamento parcial dos assistidos, ao invés de financiamento público ${ }^{33}$.

A separação de funções entre financiamento e prestação implica na precificação dos serviços de 
Tabela 3. Cumprimento da Meta 6 do ODM: alcançar até 2010 acesso a tratamento de doenças. infectocontagiosas

\begin{tabular}{|c|c|c|c|c|c|c|c|c|c|c|}
\hline \multicolumn{2}{|l|}{ Indicador } & \multirow{2}{*}{$\begin{array}{c}\text { HIV entre } \\
15 \text { e } 19 \text { anos } \\
(\%) \\
\text { Interromper } \\
\text { em } 2015 \mathrm{e} \\
\text { reverter a } \\
\text { incidência }\end{array}$} & \multicolumn{2}{|c|}{$\begin{array}{c}\text { Pop.c/ HIV c/ } \\
\text { acesso a ARV } \\
(\%)\end{array}$} & \multirow{2}{*}{$\begin{array}{c}\begin{array}{c}\text { Incidência } \\
\text { de malária } \\
\text { (por } 100 \\
\text { mil) }\end{array} \\
\begin{array}{r}\text { Interrom } \\
\text { da incidên }\end{array} \\
(\end{array}$} & \multirow{2}{*}{$\begin{array}{c}\begin{array}{c}\text { Incidência } \\
\text { de TB } \\
\text { (por } 100 \\
\text { mil) }\end{array} \\
\text { per em } 2015 \\
\text { cia em de m } \\
\text { Dados de TB }\end{array}$} & \multirow{2}{*}{\multicolumn{2}{|c|}{$\begin{array}{cc}\begin{array}{c}\text { Prevalência } \\
\text { de TB } \\
\text { (por 100 } \\
\text { mil) }\end{array} & \begin{array}{c}\text { Mortes } \\
\text { por TB } \\
\text { (por } 100 \\
\text { mil) }\end{array} \\
\end{array}$}} & \multicolumn{2}{|c|}{$\begin{array}{c}\% \text { casos TB } \\
\text { detectados e } \\
\text { curados por } \\
\text { DOTS }^{*}\end{array}$} \\
\hline Metas & Anos & & $\begin{array}{c}\text { Data } \\
\text { dos } \\
\text { Dados }\end{array}$ & $\begin{array}{c}100 \% \text { de } \\
\text { tratamento } \\
\text { HIV/Aids }\end{array}$ & & & & & D & C \\
\hline \multirow[t]{7}{*}{ ARG } & 1990 & 0,115 & & & & & 95 & 4,2 & 63 & $\ldots$ \\
\hline & 1995 & 0,212 & & & & & 69 & 3,3 & 79 & 12 \\
\hline & 2000 & 0,302 & & & 4,75 & 37 & 55 & 2,3 & 79 & 47 \\
\hline & 2005 & 0,385 & 2009 & 80,5 & 2,58 & 31 & 45 & 1,9 & 82 & 46 \\
\hline & 2010 & 0,446 & 2010 & 77,2 & 0,14 & 20 & 37 & 1,4 & 66 & 45 \\
\hline & 2014 & 0,474 & 2011 & 78,6 & 0 & 25 & 31 & 1,4 & 89 & .. \\
\hline & Var\% & 312,2 & & $-2,4$ & $-97,1$ & $-32,4$ & $-67,4$ & $-66,7$ & 41,3 & 275,0 \\
\hline \multirow[t]{7}{*}{ BRA } & 1990 & - & & & & & 129 & 5,4 & 60 & ... \\
\hline & 1995 & - & & & & & 98 & 5,3 & 79 & 17 \\
\hline & 2000 & - & & 84,1 & 62,79 & 51 & 77 & 4,4 & 74 & 71 \\
\hline & 2005 & - & 2009 & 90,7 & 38,35 & 50 & 60 & 3,1 & 85 & 72 \\
\hline & 2010 & - & 2010 & 95,0 & 19,04 & 44 & 56 & 2,8 & 81 & 72 \\
\hline & 2014 & - & 2011 & & 7,52 & 42 & 57 & 2,2 & 82 & .. \\
\hline & Var\% & - & & 13,0 & $-69,7$ & $-13,7$ & $-27,3$ & $-36,4$ & 9,5 & 323,5 \\
\hline \multirow[t]{7}{*}{$\mathrm{CHI}$} & 1990 & 0,154 & & & & & 67 & 5,8 & 90 & $\ldots$ \\
\hline & 1995 & 0,171 & & & & & 42 & 3,5 & 86 & 79 \\
\hline & 2000 & 0,193 & & & - & 23 & 30 & 1,9 & 84 & 82 \\
\hline & 2005 & 0,227 & 2009 & 59,3 & - & 18 & 21 & 1,5 & 91 & 83 \\
\hline & 2010 & 0,266 & 2010 & 63,7 & - & 16 & 22 & 1,6 & 84 & 71 \\
\hline & 2015 & 0,289 & 2011 & 66,4 & - & 17 & 19 & 1,2 & 88 &.. \\
\hline & Var\% & 87,7 & & & & $-26,1$ & $-71,6$ & $-79,3$ & 79,3 & $-2,2$ \\
\hline \multirow[t]{7}{*}{$\mathrm{COL}$} & 1990 & 0,077 & & & & & 81 & 5 & 70 & $\ldots$ \\
\hline & 1995 & 0,28 & & & & & 80 & 4 & 56 & $\ldots$ \\
\hline & 2000 & 0,511 & & & 35,71 & 36 & 65 & 3,2 & 68 & 80 \\
\hline & 2005 & 0,476 & 2009 & 25,2 & 26,34 & 30 & 55 & 2,4 & 62 & 71 \\
\hline & 2010 & 0,427 & 2010 & 45,1 & 24,48 & 31 & 48 & 2 & 71 & 74 \\
\hline & 2014 & 0,403 & 2011 & 45,8 & 9,54 & 31 & 43 & 1,6 & 75 & .. \\
\hline & Var\% & 423,4 & & 81,7 & $-73,3$ & $-13,9$ & $-46,9$ & $-68,0$ & 7,1 & $-7,5$ \\
\hline
\end{tabular}

saúde, transforma-os em mercadoria, produzida e demandada de forma concorrencial. Embora os gastos com saúde tenham aumentado nos países analisados, a participação dos gastos públicos caiu em todos os países na segunda fase, quando o neoliberalismo se espalha na região. Como destacam Dardot e Laval ${ }^{16}$, nos sistemas neoliberais não se exclui o Estado, mas este é chamado a um papel que não apenas aceita a lógica do mercado, mas funciona procurando imitá-la. A concorrência, antes relacionada ao comportamento das empresas, no neoliberalismo passa a ser característica do comportamento dos indivíduos, que funcionam como empresa de si mesmos, ao planejar e concorrer no mercado de trabalho para acessar o plano de saúde que mais lhe convém, e do Estado, que passa a se comportar como empresa, concorrendo como os demais prestadores de serviços. Assim, observa-se a transformação da ação pública, tornando o Estado uma esfera que também é regida por regras de concorrência e submetida a exigências de eficácia semelhantes àquelas que sujeitam as empresas privadas (p.272 ${ }^{16}$.

Até os anos 1980 predominaram os sistemas de saúde nacionais, solidários e regulados, com exceção da Colômbia que mostra uma descentra- 
Tabela 3. Cumprimento da Meta 6 do ODM: alcançar até 2010 acesso a tratamento de doenças. infectocontagiosas

\begin{tabular}{|c|c|c|c|c|c|c|c|c|c|c|}
\hline \multicolumn{2}{|l|}{ Indicador } & \multirow{2}{*}{$\begin{array}{c}\text { HIV entre } \\
15 \text { e } 19 \text { anos } \\
(\%)\end{array}$} & \multicolumn{2}{|c|}{$\begin{array}{c}\text { Pop.c/ HIV c/ } \\
\text { acesso a ARV } \\
(\%)\end{array}$} & \multirow{2}{*}{$\begin{array}{c}\begin{array}{c}\text { Incidência } \\
\text { de malária } \\
\text { (por } 100 \\
\text { mil) }\end{array} \\
\begin{array}{c}\text { Interrom } \\
\text { da incidên }\end{array} \\
\text { (I }\end{array}$} & $\begin{array}{c}\text { Incidência } \\
\text { de TB } \\
\text { (por } 100 \\
\text { mil) } \\
\end{array}$ & $\begin{array}{l}\text { Prevalência } \\
\text { de TB } \\
\text { (por } 100 \\
\text { mil) }\end{array}$ & \multirow[t]{2}{*}{$\begin{array}{c}\text { Mortes } \\
\text { por TB } \\
\text { (por 100 } \\
\text { mil) }\end{array}$} & \multicolumn{2}{|c|}{$\begin{array}{c}\% \text { casos TB } \\
\text { detectados e } \\
\text { curados por } \\
\text { DOTS }\end{array}$} \\
\hline Metas & Anos & & $\begin{array}{c}\text { Data } \\
\text { dos } \\
\text { Dados }\end{array}$ & $\begin{array}{c}100 \% \text { de } \\
\text { tratamento } \\
\text { HIV/Aids }\end{array}$ & & $\begin{array}{l}\text { per em } 2015 \\
\text { cia em de ma } \\
\text { Cados de TB }\end{array}$ & $\begin{array}{l}\text { e começar a r } \\
\text { lária e outras } \\
\text { - 1990-2013) }\end{array}$ & & D & C \\
\hline \multirow[t]{7}{*}{ CUB } & 1990 & 0,013 & & & & & 57 & 0,6 & 21 & \\
\hline & 1995 & 0,023 & & & & & 30 & 0,9 & 76 & 90 \\
\hline & 2000 & 0,04 & & & & 12 & 18 & 0,4 & 82 & 93 \\
\hline & 2005 & 0,076 & 2009 & 95,0 & - & 7,9 & 13 & 0,3 & 74 & 90 \\
\hline & 2010 & 0,148 & 2010 & 95,0 & - & 8,4 & 14 & 0,4 & 79 & 90 \\
\hline & 2015 & 0,25 & 2011 & 95,9 & - & 7 & 13 & 0,3 & 72 & $\ldots$ \\
\hline & Var\% & 1823,1 & & 0 & - & $-41,7$ & $-77,2$ & $-50,0$ & 242,9 & 0 \\
\hline \multirow[t]{7}{*}{ MÉX } & 1990 & 0,657 & & & & & 131 & 7,8 & 27 & $\ldots$ \\
\hline & 1995 & 0,641 & & & & & 79 & 5,5 & 28 & 75 \\
\hline & 2000 & 0,498 & & & 6,55 & 23 & 44 & 3,3 & 62 & 76 \\
\hline & 2005 & 0,352 & 2009 & 80,3 & 2,46 & 21 & 29 & 2,5 & 80 & 74 \\
\hline & 2010 & 0,262 & 2010 & 79,4 & 0,94 & 21 & 27 & 2,2 & 83 & 82 \\
\hline & 2014 & 0,23 & 2011 & 83,6 & 0,37 & 21 & 26 & 1,8 & 82 & $\cdots$ \\
\hline & Var\% & $-65,0$ & & 4,1 & $-94,4$ & $-8,7$ & $-80,1$ & $-76,9$ & 203,7 & 9,3 \\
\hline \multirow[t]{7}{*}{ PER } & 1990 & 0,583 & & & & & 336 & 34 & 71 & $\ldots$ \\
\hline & 1995 & 0,575 & & & & & 316 & 26 & 79 & 83 \\
\hline & 2000 & 0,559 & & & 23,51 & 186 & 243 & 14 & 80 & 90 \\
\hline & 2005 & 0,488 & 2009 & 46,2 & 26,29 & 153 & 197 & 9,7 & 81 & 91 \\
\hline & 2010 & 0,401 & 2010 & 43,7 & 9,7 & 132 & 174 & 8,5 & 80 & 68 \\
\hline & 2014 & 0,356 & 2011 & 60,5 & 19,67 & 119 & 164 & 7,7 & 79 & $\ldots$ \\
\hline & Var\% & $-38,9$ & & 31,0 & $-16,3$ & $-36,0$ & $-51,2$ & $-77,4$ & 11,3 & $-18,1$ \\
\hline
\end{tabular}

Website/http://mdgs.un.org/unsd/mdg/Data.aspx. Legenda: TB - tuberculose; ARV - antirretroviral; DOTS - Tratamento Diretamente Observado de Curta Duração; C - casos curados; D - cases detectados.

Fonte: CEPALStat - https://estadisticas.cepal.org/cepalstat/portada.html, Global Health Observatory data repository https://www.who.int/ data/gho e MDG Indicators.

lização e diversificação de fontes não públicas de saúde mais cedo e de forma mais ampla ${ }^{33}$. Nos demais, é nos anos 1990 que avança a lógica de mercado, enquanto nos anos 2000 em diante observam-se alguns retrocessos desta lógica ainda mantendo, porém, um papel importante do setor privado e da concorrência na prestação de serviços de saúde s11,13,29. $^{1}$.

Finalmente, a focalização dos serviços básicos e a entrega a governos locais, entidades não governamentais e à iniciativa privada, ao fragmentar a prestação de serviços, amplia a segmentação da população e leva a deseconomias por ineficiência alocativa. Esse tratamento diferenciado é ampliado pela diferenciação que se faz da população alvo de pacotes de serviços distintos, por formas de financiamento diferentes e por cober- turas diversas dos serviços de saúde ${ }^{29}$. Todas essas diferenciações caracterizaram as reformas de saúde e justificaram a queda dos gastos públicos em saúde, tornando precária a prestação de serviços de saúde, principalmente para a população mais pobre.

Esta estratégia, contudo, vai contra o que está explícito na meta 3.8 dos ODS, que visa atingir a cobertura universal de saúde, incluindo a proteção do risco financeiro, o acesso a serviços de saúde essenciais de qualidade e o acesso a medicamentos e vacinas essenciais seguros, eficazes, de qualidade e a preços acessíveis para todos ${ }^{21}$. Daí o porquê de seu potencial de mudanças estruturantes ter sido questionado ${ }^{3}$.

A estratégia dos organismos internacionais, na direção do modelo de CUS foi insuficien- 
te para garantir o cumprimento das metas dos ODM. Nenhuma delas foi atingida completamente pelos países e algumas não foram cumpridas por nenhum país. Chama a atenção em especial a mortalidade materna alta, a gravidez na adolescência, a baixa cobertura pré-natal, presentes em alguns países da região, sintetizando os desafios existentes para melhorar a saúde sexual e reprodutiva das mulheres ${ }^{35}$. Progressos foram obtidos nos nascimentos assistidos, na redução da incidência de malária e incidência e prevalência de tuberculose, mas só Brasil e Cuba chegaram perto da meta de atendimento integral à população com HIV/Aids.

Os indicadores que se referem à saúde da mulher, como a RMM e a gravidez na adolescência sinalizam para um efeito perverso dos sistemas de saúde que é a desassistência ou baixa qualidade do cuidado ofertado à saúde reprodutiva. Os efeitos no longo prazo atingem as famílias e a economia como um todo na medida em que mortes maternas em geral deixam crianças e idosos desprovidos de cuidado. Também a morte de mulheres afeta a oferta de cuidados familiares e mais ainda, a renda familiar ${ }^{35}$.

Este artigo tem como limitações o seu caráter descritivo na análise dos dados o que não permite afirmar relações causais mais fortes entre o período de dados analisado e as mudanças ocorridas em cada país. Todavia, a análise das características das reformas, conforme mostra o Quadro 1, e da evolução do neoliberalismo mostra que, no primeiro período analisado (até 1990), predomina o papel do Estado no provimento e regulamentação dos sistemas de saúde. O segundo período (1990-2000) mostra uma lógica de mudanças mais concorrencial obedecendo à lógica do mercado, enquanto o último período (20012015), apesar de manter o estímulo à concorrência, mostra a introdução de programas de lógica pública e comunitária. A análise das reformas, além disso, permite mostrar que o alcance de resultados assistenciais tem uma relação estreita com a capacidade dos Estados nacionais de intervirem de forma coordenada nos determinantes sociais, e está relacionado ao aumento dos gastos públicos em saúde e à capacidade de governança e coordenação interfederativas internas nos países. Em outras palavras, as desigualdades no acesso aos recursos de saúde precisam ser tratadas por meio de regulamentações internacionais e de jurisdições nacionais e sistemas de saúde $\mathrm{de}^{34,36}$. A trajetória dos sistemas de saúde com forte descentralização e fragmentação e oscilações no financiamento, em uma região com forte desigualdade social sinaliza para a necessidade de novos rumos nas reformas, em prol do fortalecimento dos sistemas públicos de saúde.

\section{Conclusões}

Os grandes desafios dos sistemas de saúde seguem sendo: as gritantes diferenças nos resultados em saúde decorrentes das iniquidades socioeconômicas e nas diferenças na qualidade dos serviços públicos e privados; a fragmentação da organização da prestação de serviços e a segmentação do financiamento que tolera a existência de acesso a carteiras de serviços compatíveis com a capacidade contributiva dos segmentos populacionais; a baixa regulação do setor privado em relação à oferta de serviços, custos e lucros; a dificuldade de atender as necessidades das populações em momento de transição demográfica, epidemiológica e de movimentos contestatórios, entre outros.

As reformas dos sistemas de saúde da ALC interferiram nos países tanto nos aspectos sanitários quanto na trajetória das políticas de saúde, aprofundando desigualdades sociais e econômicas e deteriorando as condições de vida das populações. Tal deterioração, agravada pelas crises econômicas (1980-90 e 2008 e pós-pandemia COVID-19), dificultará o alcance dos ODS e produzirá ambiente propício para o aparecimento e ressurgimento de enfermidades e agravamento dos problemas de saúde pública, requerendo papel diferente do sistema público de saúde, mais amplo e integrado, sob pena de reversão grave na situação de saúde da população. 


\section{Colaboradores}

LBD Göttems e LP Camilo trabalharam na concepção, no delineamento, na coleta, na análise, na interpretação dos dados e na redação do artigo. MLR Mollo trabalhou na redação do artigo e na sua revisão crítica. C Mavrot trabalhou na redação do artigo e na aprovação da versão a ser publicada.

\section{Referências}

1. United Nations. The Millennium Development Goals Report 2015. [cited 2021 Nov 04]. Available from: https://www.un.org/millenniumgoals/2015_MDG_Report/pdf/MDG\%202015\%20rev\%20(July\%201).pdf

2. Gallo E, Setti AFF. Território, intersetorialidade e escalas: requisitos para a efetividade dos Objetivos de Desenvolvimento Sustentável. Cien Saude Colet 2014; 19(11):4383-4396.

3. Buss PM, Magalhães DP, Setti AFF, Gallo E, Franco Neto FA, Machado JMH, Buss DF. Saúde na Agenda de Desenvolvimento pós-2015 das Nações Unidas. Cad Saude Publica 2014; 30(12):2555-2570.

4. Giovanella L, Mendoza-Ruiz A, Pilar ACA, Rosa MC, Martins GB, Santos IS, Silva DB, Vieira JML, Castro VCG, Silva PO, Machado CV. Universal health system and universal health coverage: assumptions and strategies. Cien Saude Colet 2018; 23(6):1763-1776.

5. Laurell AE. Políticas de saúde em conflito: seguro contra os sistemas públicos universais. Rev Latino-Am Enfermagem 2016; 24: e2668.

6. Bértola L, Ocampo JA. Desarrollo, vaivenes y desigualdad una historia económica de América Latina desde la independencia. Secretaría General Iberoamerica. Agencia Española de Cooperacion Internacional para el Desarrollo; 2010.

7. Birn A, Nervi L, Siqueira E. Neoliberalism Redux: The Global Health Policy Agenda and the Politics of Cooptation in Latin America and Beyond. Develop Change 2016; 47(4):734-759.

8. Comisión Económica Para América Latina Y El Caribe. Panorama social de América Latina, Santiago, 1996 [acceso 2021 Nov 04]. Disponible en: https://www. cepal.org/es/publicaciones/1253-panorama-social-america-latina-1996.

9. Pereira JMM, organizador. A demolição de direitos: um exame das políticas do Banco Mundial para a educação e a saúde (1980-2013). Organização de João Márcio Mendes Pereira e Marcela Pronko. Rio de Janeiro: Escola Politécnica de Saúde Joaquim Venâncio; 2014.

10. Pereira JMM. Banco Mundial, reforma dos Estados e ajuste das políticas sociais na América Latina. Cien Saude Colet 2018; 23(7):2187-2196.

11. Chodor T. Neoliberal hegemony and the pink tide in Latin America: Breaking up with TINA? Berlin: Springer; 2014.

12. World Bank. World Development Report 1993: Investing in Health. New York: Oxford University Press; 1993. [cited 2021 Nov 04]. Available from: https:// openknowledge.worldbank.org/handle/10986/5976

13. Levcovitz E, Couto MHC. Sistemas de saúde na América Latina no século XXI. [acessado 2021 nov 10]. Disponível em: http://capacidadeshumanas.org/oichsitev3/wp-content/uploads/2019/02/4-Sistemas-desa\%C3\%BAde-na-Am\%C3\%A9rica-Latina-no-s\%C3\%A9culo-XXI.pdf-

14. Alvarez MH. Neoliberalismo em salud: desarrollos, supuestos y alternativas. In: La falacia neoliberal: criticas y alternativas. Bogotá: Universidad Naional de Colombia; 2003.

15. Machado CV. Políticas de Saúde na Argentina, Brasil e México: diferentes caminhos, muitos desafios. Cien Saude Colet 2018; 23(7):2197-2212. 
16. Dardot P, Laval C. Uma nova razão do mundo: ensaio sobre a sociedade neoliberal. São Paulo: Boitempo; 2016.

17. Almeida PF, Oliveira SC, Giovanella L. Network integration and care coordination: the case of Chile's health system. Cien Saude Colet 2018; 23(7):2213-2227.

18. Levino A, Carvalho EF. Análise comparativa dos sistemas de saúde da tríplice fronteira: Brasil/Colômbia/ Peru. Rev Panam Salud Publica 2011; 30(5):490-500.

19. López-Arellano O, Jarillo-Soto EC. Implicaciones de las reformas de salud en méxico. Cad Saude Publica 2017a; 33(Supl. 2):e00087416

20. López-Arellano O, Jarillo-Soto EC. La reforma neoliberal de un sistema de salud: evidencia del caso mexicano Cad Saude Publica 2017b; 33(Supl. 2):e00087416

21. Madureira PSP. Sistema de Saúde Cubano [dissertação]. Coimbra (Portugal): Faculdade de Medicina da Universidade de Coimbra; 2010

22. Osa JA. Um olhar para a saúde pública cubana. Estudos Avançados 2011; 25(72): 89-96.

23. Bermudez AV, Luque FC, Reyes CS. Flexibilización laboral en el Perú y reformas de la protección social asociadas: un balance tras 20 años. Serie Políticas Sociales, no 175, (LC/L.3444). Santiago de Chile: Comisión Económica para América Latina y el Caribe (CEPAL)/Agencia Alemana de Cooperación Internacional (GTZ). [acceso 2021 Nov 08]. Disponible en: https://repositorio.cepal.org/bitstream/handle/11362/ 6188/1/S1100960_es.pdf_

24. Mesa-Lago C. Sistemas de protección social en América Latina y el Caribe: Cuba. 2012. [acceso $2021 \mathrm{Nov}$ 10]. Disponible en: https://repositorio.cepal.org/bitstream/handle/11362/4034/LCw510_es.pdf?sequen$\mathrm{ce}=1$ \&isAllowed $=\mathrm{y}_{\text {_ }}$

25. Lavigne M. Sistemas de protección social en América Latina y el Caribe: Perú. [acceso 2021 Nov 10]. Disponible en: https://repositorio.cepal.org/bitstream/ handle/11362/4047/S2013058_es.pdf?sequence=1\&isAllowed $=\mathrm{y}$

26. Mesa-Lago C. Las reformas de salud en América Latina y el Caribe: su impacto en los principios de la seguridad social. Comisión Económica para América Latina y el Caribe (CEPAL). Documentos de proyetos. 2005 [acceso 2021 Nov 10]. Disponible en: https:// repositorio.cepal.org/bitstream/handle/11362/3888/ S2005077_es.pdf

27. Sánchez-Moreno F. El sistema nacional de salud en el Perú. Rev Peru Med Exp Salud Publica 2014; 31(4):747-753.

28. Gómez-García CA, Builes-Velásquez A. (2018) El derecho fundamental a la salud y la política de acceso al sistema: una mirada desde la Ley Estatutaria 1751 del año 2015. Rev Facultad de Derecho y Ciencias Políticas 48(128):135-167.
29. Atun R, Andrade LOM, Almeida G, Cotlear D, Dmytraczenko T, Frenz P, Garcia T, Garcia P, GómezDantés O, Knaul FM, Muntaner C, Paula JB, Rígoli F, Serrate PCF, Wagsta A. Health-system reform and universal health coverage in Latin America. Lancet 2015; 385: 1230-1247.

30. Pan American Health Organization (PAHO). Health Conditions in the Americas 1981-1984.Vol. 2. Washington: PAHO; 1986. [cited 2021 Nov 03]. Available from: http://iris.paho.org/xmlui/handle/123456789/28374

31. Pan American Health Organization (PAHO). Health Conditions In The Americas. Vol. 2. Washington: Paho; 1990. [cited 2021 Nov 03]. Available from: http://iris.paho.org/xmlui/handle/123456789/28372

32. Jaramillo I. Evaluación de la descentralización de la salud y la reforma de la Seguridad Social en Colombia. Gac Sanit 2002;16(1):48-53

33. Lima LD. Crisis y neoliberalismo: sistemas universales de salud en américa latina. Cad Saude Publica 2017; 33(Supl. 2):e00047517

34. Göttems LBD, Mollo MLR. Neoliberalismo na América Latina: efeitos nas reformas dos sistemas de saúde. Rev Saude Publica 2020; 54:74.

35. Comisión Económica Para América Latina Y El Caribe (CEPAL). Horizontes 2030: la igualdad en el centro del desarrollo sostenible (LC/G.2660/Rev.1). Santiago: CEPAL; 2016.

36. Niessen LW, Mohan D, Akuoku JK, Mirelman AJ, Ahmed S, Koehlmoos TP, Trujillo A, Khan J, Peters DH. Tackling socioeconomic inequalities and noncommunicable diseases in low-income and middle -income countries under the Sustainable Development agenda. Lancet 2018; 391:2036-2046.

Artigo apresentado em 20/11/2020

Aprovado em 24/05/2021

Versão final apresentada em 26/05/2021

Editores-chefes: Romeu Gomes, Antônio Augusto Moura da Silva 